\title{
Long-term results of surgical treatment in benign bronchoesophageal fistula
}

\author{
Hong Kwan Kim, MD, Yong Soo Choi, MD, Kwhanmien Kim, MD, Jhingook Kim, MD, and Young Mog Shim, MD
}

Objectives: Benign bronchoesophageal fistula is a rare complication resulting from various diseases. The objectives of the study are to report our surgical experience in patients with benign bronchoesophageal fistulas and to evaluate the long-term results of surgical management.

Methods: Between 1995 and 2005, a total of 14 patients (mean age, 52.5 years; male/female $=6: 8$ ) underwent operations for benign bronchoesophageal fistulas. The etiology of the fistulas included traction esophageal diverticula in 7 patients and tuberculous lymphadenopathy in 6. Primary repair of the fistula was performed in all patients and a concomitant lobectomy in 6.

Results: There was no in-hospital mortality. Postoperative complications occurred in 1 patient (empyema resulting from a leakage at the repair site). Postoperative esophagography revealed a leakage at the repair site in 1 patient. There was 1 late death with a mean follow-up of 43.9 months. The cause of death was aspiration pneumonia resulting from recurrent fistula. Two of 8 patients who did not undergo a lobectomy had persistent bronchiectasis, whereas none of those who underwent a concomitant lobectomy had any recurrent pneumonia or bronchiectasis. The overall survival was $100 \%$ at 5 years and the mean survival time was 124 months $(95 \%$ confidence interval, 106.5-142.2 months).

Conclusions: We conclude from these data that early detection and definitive surgical repair of benign bronchoesophageal fistulas can yield high success rates with excellent early outcomes and good long-term results.

From the Department of Thoracic and Cardiovascular Surgery, Samsung Medical Center, Seoul, Republic of Korea.

Received for publication Dec 31, 2006; revisions received March 6, 2007; accepted for publication April 11, 2007.

Address for reprints: Yong Mog Shim, MD, Department of Thoracic and Cardiovascular Surgery, Samsung Medical Center, 50 Ilwon-Dong, Gangnam gu, Seoul 135-710, Republic of Korea (E-mail: ymshim@smc. samsung.co.kr).

J Thorac Cardiovasc Surg 2007;134:411-4

0022-5223/\$32.00

Copyright $\odot 2007$ by The American Association for Thoracic Surgery

doi:10.1016/j.jtcvs.2007.04.030
$\mathrm{M}$ ost bronchoesophageal fistulas (BEFs) result from locally advanced bronchogenic or esophageal malignant disease, and BEFs of benign origin are rare. Because the clinical course tends to be both insidious and nonspecific in patients with benign BEFs, it is difficult to make a correct diagnosis before BEFs develop secondary complications such as aspiration pneumonia or chronic bronchitis. Therefore, it is important to suspect the possibility of BEF when a patient presents with unexplained persistent cough. Once a diagnosis of BEF is appropriately established, surgical treatment is considered definitive, leading to successful outcomes. However, there have been few reports concerning surgical treatment of BEFs and the long-term results. The objectives of this study are to report our surgical experience in patients with benign BEFs and to evaluate the long-term results of surgical management in this potentially devastating disease.

\section{Patients and Methods \\ Patients Selection}

Samsung Medical Center is one of the largest tertiary referral hospitals in Korea, with approximately 1500 general thoracic operations annually. Between January 1995 and December 2005, a total of 16 consecutive patients underwent operations for BEFs at our institution. Of those, 2 patients had BEF resulting from esophageal cancer and these were excluded from the study. The remaining 14 patients had benign etiologies. The medical 


\section{Abbreviations and Acronyms}

$\mathrm{BEF}=$ bronchoesophageal fistula

records were retrospectively reviewed to evaluate the clinical characteristics, operative findings, postoperative courses, and longterm results. This study was reviewed and approved by the Institutional Review Board of the Sungkyunkwan University.

\section{Clinical Characteristics}

Mean age was $52.5 \pm 14.2$ years (range, 26-78 years). Six patients were male and 8 female. The etiology of the BEFs was primary mediastinal inflammation in all patients except in 1 case of unknown cause. Seven patients had traction diverticula, which were mostly caused by mediastinal granulomatous inflammation such as tuberculosis. In 6 patients, tuberculous mediastinal lymphadenopathy was identified, on the basis of a history of tuberculosis, operative findings (enlarged mediastinal lymph nodes), or pathologic findings (caseating necrosis).

Most patients had a severe cough exacerbated by swallowing liquids $(\mathrm{n}=5)$ and also reported unexplained productive cough $(\mathrm{n}=5)$, hemoptysis $(\mathrm{n}=3)$, and chest pain $(\mathrm{n}=1)$. Eleven $(78.6 \%)$ patients had persistent symptoms that remained undiagnosed for variable periods ( 3 months-20 years). Six (42.9\%) patients had initially sought medical attention elsewhere and were given presumptive diagnoses such as pneumonia $(\mathrm{n}=2)$, bronchiectasis $(\mathrm{n}=1)$, pulmonary tuberculosis $(\mathrm{n}=1)$, lung cancer $(\mathrm{n}=1)$, and gastroesophageal reflux $(\mathrm{n}=1)$. All patients underwent at least one or two diagnostic tests, including esophagography, esophagoscopy, examination with a flexible bronchoscope, and computed tomography to confirm the presence of fistula and to evaluate its anatomic features. Esophagography confirmed BEFs in all cases, whereas some of the esophagoscopic $(n=2)$ and bronchoscopic findings $(n=6)$ failed to reveal BEFs. The sensitivity of esophagography, esophagoscopy, and bronchoscopy was $100 \%, 75 \%$, and $40 \%$, respectively (Table 1$)$. Ten patients $(71.4 \%)$ had complications resulting from fistulas, such as aspiration pneumonia $(n=6)$ and bronchiectasis $(n=4)$. The clinical characteristics of these patients are shown in Table 2.

\section{Surgical Technique}

All operations were performed on an elective basis to stabilize the patients, with adequate nutritional support and intensive control of

TABLE 1. Comparison of the sensitivity among esophagography, esophagoscopy, and bronchoscopy in detecting BEF

\begin{tabular}{lccc}
\hline & Esophagography & Esophagoscopy & Bronchoscopy \\
\hline $\begin{array}{l}\text { No. of patients } \\
\quad \text { examined }\end{array}$ & 14 & 8 & 10 \\
$\begin{array}{l}\text { True positive (No.) } \\
\text { False positive (No.) }\end{array}$ & 14 & 6 & 4 \\
Sensitivity (\%) & 0 & 2 & 6 \\
\hline BEF Bron & 100 & 75 & 40 \\
\hline
\end{tabular}

$B E F$, Bronchoesophageal fistula.
TABLE 2. Patient characteristics

\begin{tabular}{lc}
\hline Characteristic & No. (\%) \\
\hline Age (y, mean \pm SD) & $52.5 \pm 14.2$ \\
Gender & \\
Male & $6(43)$ \\
Female & $8(57)$ \\
Etiology of fistula & \\
Esophageal diverticulum & $7(50)$ \\
Tuberculous lymphadenopathy & $6(43)$ \\
Unknown & $1(7)$ \\
Secondary complication & \\
Pneumonia & $6(43)$ \\
Bronchiectasis & $4(29)$ \\
Initial misdiagnosis & \\
Pneumonia & $2(14)$ \\
Bronchiectasis & $1(7)$ \\
Pulmonary tuberculosis & $1(7)$ \\
Lung cancer & $1(7)$ \\
Gastroesophageal reflux & $1(7)$ \\
\hline
\end{tabular}

infection preoperatively. Total parenteral nutrition $(n=2)$ and feeding gastrostomy $(n=2)$ were used in 4 patients. Every effort was made to eliminate the fistulas and to preserve the physiologic passage in a single stage. All operations were performed through a right posterolateral thoracotomy except in 1 case in which a left lower lobectomy was planned. After the fistula was carefully exposed and divided, the bronchus was primarily repaired with interrupted 3-0 or 4-0 silk or Vicryl (Ethicon, Inc, Somerville, NJ) sutures and the esophagus was closed with 2 layers of 3-0 or 4-0 silk sutures. A surgical stapling device was used in 9 patients. A pedicled vascularized buttress using the parietal pleura was interposed between the bronchus and the esophagus in 3 patients. In the remaining 11 patients, a pedicled buttress using viable tissue was not used. Six $(42.9 \%)$ patients underwent a concomitant lobectomy, including right lower lobectomy in 3, right middle lobectomy in 1 , right middle and lower lobectomy in 1 , and left lower lobectomy in 1 .

\section{Anatomy of Fistula}

Operative findings showed that the course of the fistula tract was diverse (Table 3 ). The most common tract (6/14 patients) was from

TABLE 3. The course of the fistulous tract in BEF

\begin{tabular}{ll}
\hline & No. \\
\hline Bronchus & \\
Right lower lobe & 6 \\
Bronchus intermedius & 3 \\
Right main stem & 2 \\
Right middle lobe & 1 \\
Left lower lobe & 2 \\
Esophagus & \\
Midthoracic & 8 \\
Lower thoracic & 6 \\
\hline
\end{tabular}

$B E F$, Bronchoesophageal fistula. 
the right bronchial tree to the distal esophagus (below $28 \mathrm{~cm}$ from the incisors). The site of airway entry entailed the right lower lobe bronchus in 6 patients, the bronchus intermedius in 3, the right main stem bronchus in 2, the right middle lobe bronchus in 1 , and the left lower lobe bronchus in 2 . The fistula drained into the distal esophagus in 8 patients and midesophagus in 6 . The diameter of the fistula was documented in 10 patients and ranged from $5 \mathrm{~mm}$ to $3 \mathrm{~cm}(14.0 \pm 8.1 \mathrm{~mm})$. The length of the fistula was recorded in 6 patients and ranged from $10 \mathrm{~mm}$ to 4 $\mathrm{cm}(24.2 \pm 11.1 \mathrm{~mm})$.

\section{Postoperative Management and Follow-up}

All but 1 patient were extubated immediately after the operation. The patient who received a repeat thoracotomy remained intubated for 66 hours postoperatively because of increased pulmonary secretions. Patients were reassessed by esophagography 3 or 4 days after the operation and their oral intake was resumed thereafter. Nine $(64.3 \%)$ patients were discharged home within 7 days. The median postoperative length of stay was 7 days (mean, 11.9 days; range, 5-58 days).

Follow-up was complete for all patients and the mean follow-up was $43.9 \pm 46.5$ months (range, 3-136 months). Descriptive statistics included the mean and standard deviation for continuous variables and frequencies and percentages for categorical variables. Late outcomes were analyzed with the Kaplan-Meier method.

\section{Results}

There was no in-hospital mortality. Postoperative complications occurred in 1 patient. The patient had previously undergone a right lower lobectomy for bronchiectasis, and a fistula developed between the right middle lobe bronchus and the midesophagus owing to traction esophageal diverticulum. On postoperative esophagography, she had a leakage at the repair site, which subsequently resulted in empyema. She underwent a successful repair of the leakage with an omental pedicled flap on postoperative day 7. Except in this patient, there was no leakage at the repair site on follow-up esophagography.

All patients have been followed up from 3 months to 11 years. There was 1 late death, occurring in the patient in whom a leakage developed at the repair site. The fistula recurred in this patient 9 years after the initial operation, and then she died of respiratory failure resulting from aspiration pneumonia. Except in this case, no recurrences of fistulas were observed during the follow-up period. Two of 8 patients who did not undergo a lobectomy had persistent bronchiectasis, whereas none of those who underwent a concomitant lobectomy had any recurrent pneumonia or bronchiectasis. No patients reported having dysphagia after repair of the fistula during follow-up. The overall survival was $100 \%$ at 5 years and the mean survival time was 124 months (95\% confidence interval, 106.5-142.2 months). Postoperative results are summarized in Table 4.
TABLE 4. Postoperative results after repair of BEF

\begin{tabular}{lc}
\hline Median length of stay (d) & 7 \\
Follow-up duration (mo) & $43.9 \pm 46.5$ (3-136) \\
Early morbidity & 1 \\
$\quad$ Leakage at the repair site & 1 \\
Empyema & \\
Late morbidity & 1 \\
Fistula recurrence & 2 \\
Recurrent bronchiectasis & 1 \\
Late mortality &
\end{tabular}

$B E F$, Bronchoesophageal fistula.

\section{Discussion}

$\mathrm{BEF}$ is a rare complication resulting from various diseases. It has been known that most BEFs in the adult population develop as a result of locally advanced esophageal or bronchogenic malignancy. ${ }^{1}$ A recent review of BEFs at Massachusetts General Hospital found that, in more than 40 years, a total of 228 cases were seen. ${ }^{1}$ Benign causes made up only $6 \%$ of all cases. Because a malignant fistula in itself suggests that a tumor has already invaded adjacent structures, surgical treatment has been considered only in highly selected cases. The development of self-expanding metallic stents has provided a new palliative option, and surgical bypass is currently rarely indicated. ${ }^{2}$ We had 2 cases of malignant BEFs managed surgically at our institution from 1995 to 2005, and they were excluded from our study.

However, BEFs could also develop from benign etiologies such as infection, trauma, and esophageal diverticulum, although they have been rarely reported so far ${ }^{1,3-9}$ Since Heiderich $^{10}$ first demonstrated a fistula between the right main stem bronchus and the esophagus in 1916, only about 120 cases of benign BEF have been published in the world literature. ${ }^{1}$ Even the largest single institutional series reported by Braimbridge and Keith ${ }^{11}$ in 1965 included 3 cases from their own experience and 20 cases that had been released in the literature until that time. Recently, Mangi and associates ${ }^{1}$ reviewed their surgical experience with benign BEF in 13 patients between 1960 and 2001. In our study, we encountered 14 cases of benign BEFs treated surgically from 1995 to 2005 . To our knowledge, this is one of the largest single institutional series regarding surgical repair of benign BEFs. BEFs were most commonly caused by mediastinal granulomatous inflammation such as tuberculosis. This suggests that the etiology of the BEFs in our study population might be related to the higher incidence of tuberculosis in our country.

Given the rarity of benign BEFs, it is not surprising that most patients are initially given the incorrect diagnosis, such as community-acquired pneumonia or chronic bronchitis. Mangi and associates ${ }^{1}$ reported that 5 patients were given the presumptive diagnosis of adult-onset asthma or prolonged postinfectious bronchitis among 9 patients who 
presented with a chronic cough. In our experience, 6 patients were misdiagnosed with other pulmonary diseases or gastroesophageal reflux. Making a prompt diagnosis in patients with benign BEFs is challenging because the clinical course tends to be both insidious and nonspecific. The need for definitive repair is often unnecessarily delayed, leading to the development of potentially life-threatening conditions such as aspiration pneumonia. Kim and colleagues ${ }^{8}$ showed that the duration of symptoms ranged from 5 to 30 years in their study of congenital BEF, and pulmonary resection was required owing to secondary complications in 8 of 13 patients. In our series, we observed that 8 patients had persistent symptoms for more than a year, 20 years being the longest. Ten patients had complications resulting from fistulas, and of these, 6 eventually needed a simultaneous pulmonary resection.

Accordingly, given the rarity as well as the insidious aspects of BEFs, it is of great importance to suspect the possibility of BEF when patients present with inexplicable symptoms. The initial diagnosis should be questioned in any patient who has an unexplained persistent cough and further evaluation pursued when symptoms fail to improve after established medical therapy. Although various diagnostic modalities are available and complementary to each other, barium esophagography is regarded as the most sensitive and definitive tool of diagnosis in comparison with endoscopic procedures. ${ }^{5,9}$ In our study, we noticed that some of the esophagoscopic and bronchoscopic findings failed to reveal the fistula, whereas esophagography discovered it in all cases. Although it is difficult to show the statistically significant difference owing to the limitations of sample size, esophagography was more sensitive than esophagoscopy or bronchoscopy.

Once a BEF is confirmed, early surgical intervention is considered definitive, resulting in successful outcomes. Many authors have advocated early and definitive surgical repair, and promising results have been obtained in previous studies. Mangi and associates ${ }^{1}$ reported that in-hospital mortality occurred in $2(15.4 \%)$ patients after they conducted 13 operations for BEF. In our experience, no inhospital mortality occurred and there was only 1 late death during follow-up. These excellent outcomes can be attributed in part to the relatively good preoperative functional status of the study population. Another possible reason for our success is the low threshold to perform a lobectomy when necessary. It is likely that many surgeons would attempt to save a lobe whenever possible, but we performed a concomitant lobectomy in nearly half of the patients. This might have decreased the pulmonary complication rate, which led to excellent early results. Furthermore, the longterm data showed that a concomitant lobectomy also de- creased the chances of recurrent pneumonia or bronchiectasis, which may have occurred if the lobe had been saved.

In general, it has been suggested that a pedicled vascularized buttress is required to reinforce the repair and separate the bronchus from the esophagus in the surgical treatment of BEFs. Interestingly, however, we did not use any pedicled buttress in 11 patients because we thought that it was not needed. Despite omission of this procedure, we achieved successful outcomes regarding repair of the fistula compared with the past reports. It can be speculated that interposition of viable tissue might not be routinely required so long as a diseased lobe is resected and there is no active inflammatory process.

In summary, because the BEF of benign etiology is both rare and insidious, it is difficult to make a correct and prompt diagnosis before secondary complications occur. Early detection of the fistula can be guaranteed by suspecting the possibility of it when the clinical characteristics are incompatible with the initial diagnoses. After the diagnosis is confirmed, early and definite surgical intervention with meticulous exposure of the fistula, division or resection of it, and primary repair of the bronchus and the esophagus is the treatment of choice. When a diseased lobe is identified, a concomitant lobectomy might be helpful to prevent pulmonary complications. We conducted the surgical management of benign BEF and obtained satisfactory outcomes in comparison with previous reports.

\section{References}

1. Mangi AA, Gaissert HA, Wright CD, Allan JS, Wain JC, Grillo HC, et al. Benign broncho-esophageal fistula in the adult. Ann Thorac Surg. 2002;73:911-5.

2. Low DE, Kozarek RA. Comparison of conventional and wire mesh expandable prostheses and surgical bypass in patients with malignant esophagorespiratory fistulas. Ann Thorac Surg. 1998;65:919-23.

3. Raghu G, Dillard D. Esophagobronchial fistula and mediastinal tuberculosis. Ann Thorac Surg. 1990;50:647-9.

4. Lang SJ, Mulder DG. Congenital oesophagobronchial fistula in an adult. J Thorac Cardiovasc Surg. 1987;94:788-91.

5. Cherveniakov A, Tzekov C, Grigorov GE, Cherveniakov P. Acquired benign esophago-airway fistulas. Eur J Cardiothorac Surg. 1996;10: 713-6.

6. Lado Lado FL, Golpe Gómez AG, Cabarcos Ortiz de Barrón A Antúnez López JR. Bronchoesophageal fistulae secondary to tuberculosis. Respiration. 2002;69:362-5.

7. López A, Rodríguez P, Santana N, Freixinet J. Esophagobronchial fistula caused by traction esophageal diverticulum. Eur J Cardiothorac Surg. 2003;23:128-30.

8. Kim JH, Park K-H, Sung SW, Rho JR. Congenital bronchoesophageal fistulas in adult patients. Ann Thorac Surg. 1995;60:151-5.

9. Rämö OJ, Salo JA, Mattila SP. Congenital bronchoesophageal fistula in the adult. Ann Thorac Surg. 1995;59:887-90.

10. Heiderich H. Pathologich-anatomische demonstrationen. Dtsch Med Wochenschr. 1916;42:340.

11. Braimbridge MV, Keith HI. Oesophago-bronchial fistula in the adult. Thorax. 1965;20:226-33. 